
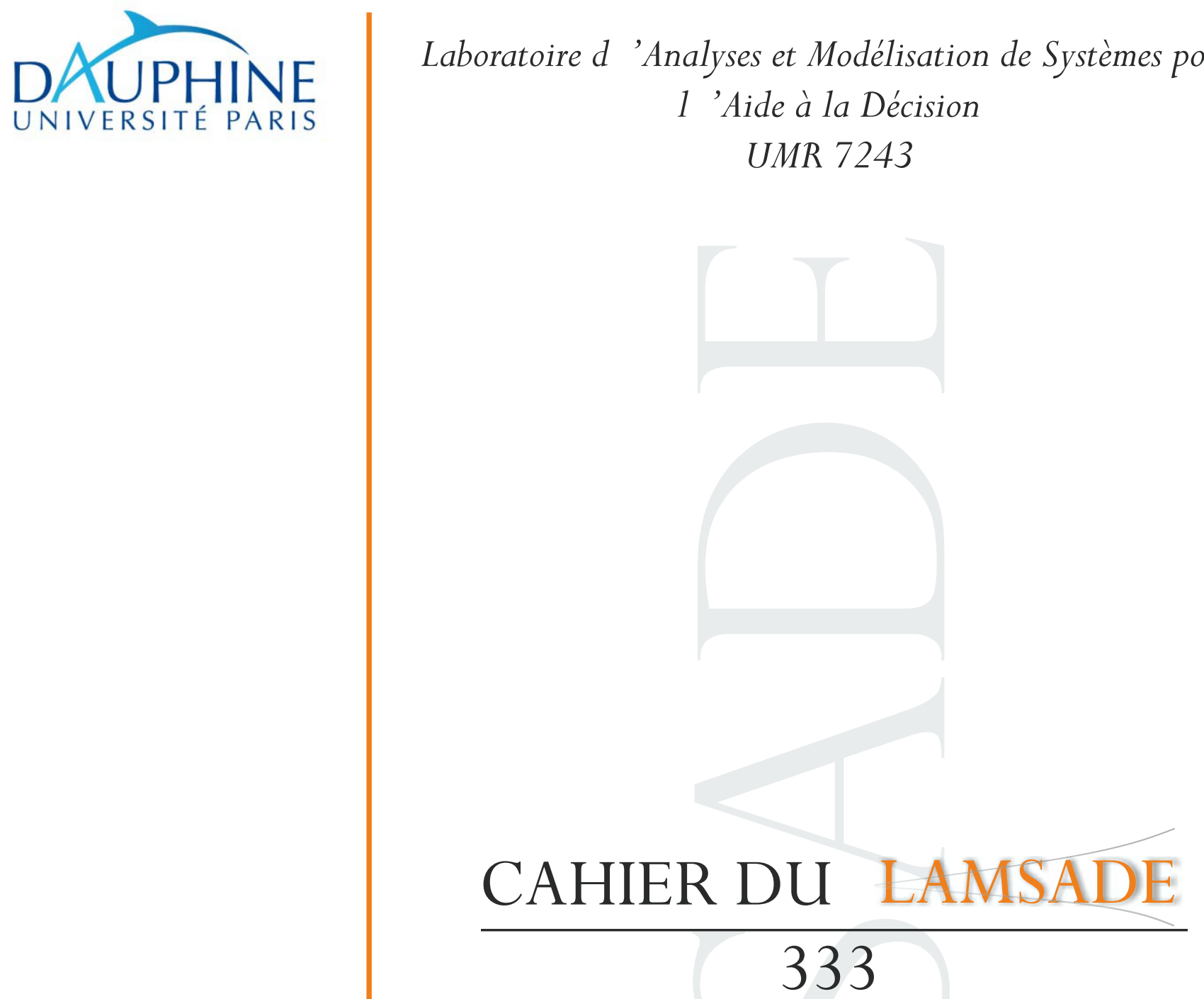

Février 2013

Robustness for Operations Research and Decision Aiding

Bernard Roy 


\title{
ROBUSTNESS FOR OPERATIONS RESEARCH AND DECISION AIDING
}

\author{
Bernard Roy \\ LAMSADE, Paris-Dauphine University \\ roy@lamsade.dauphine.fr
}

\section{Résumé}

L'article débute par une présentation succincte de ce dont s'occupe la discipline RO-AD ; deux exemples sont ensuite présentés. Le sens dans lequel le qualificatif « robuste » est utilisé en $\mathrm{RO}-\mathrm{AD}$ est précisé en section 3 où il est également expliqué pourquoi la recherche de robustesse constitue une préoccupation importante dans cette discipline. Pour bien répondre à cette préoccupation, il importe de convenablement prendre en compte deux nécessités qui sont présentées dans la section suivante. La section 5 est consacrée au schéma dans lequel se situent actuellement les réponses classiques apportées à cette préoccupation. Les deux dernières sections avant la conclusion donnent un aperçu sur des approches moins classiques.

Abstract

Robustness for Operations Research and Decision Aiding [OR-DA]

The paper begins with a succinct presentation of what OR-DA seeks to accomplish and then goes on to develop two examples. Section three, which explains why the search for robustness is a major concern in the field, details the meaning of the qualifier robust, as used in this paper. The two requirements presented in section four must be taken into consideration appropriately, if the concern is to be addressed properly. Section five deals with the framework schema wherein the standard answers to the concern can be found. The two sections before the conclusion provide an overview of less traditional approaches. 
Robustness for OR-DA is a subject that has given rise to many publications over the last several years (see for instance Aissi A., Roy B., 2010, Billaut J.C. and Al, 2008, Kouvelis T., Yu G., 1997, Newsletter 2002-2008 of the European Working Group Multiple Criteria Decision Aiding, Rosenhead J. 2001 and Roy B. 2010b). I will first present the focus of ORDA and deal with two examples that will be developed further in my paper. I will go on to explain why robustness is a major concern in OR-DA, which will lead me to underscore the two requirements that must be tackled to deal with this concern property. Then I will present the framework schema for most research and the way that the concept of a robust solution is usually addressed. In the two sections before the conclusion, I will examine the most recent research outside the schema, with other definitions of robust solutions and other types of answers (robust conclusions).

\section{What is OR-DA?}

During World War II, the Allied Forces called upon several scientists (mainly mathematicians) to enlighten the strategic and tactical decisions in the theatres of operations. This was called Operations Research (OR) (operational research at the time). After the war, the scientists transposed what they had done in the battlefield to the corporate world, thus explaining the development of linear programming, the theory of games, the queuing theory, and more. By the late forties, in England, the United States and later in France, consulting firms had the term Operations Research in their corporate name. Aside from its purely mathematical component, OR is closely linked to Decision Aiding (DA), as can be clearly seen in the name [Société Française de Recherche Opérationnelle et Aide à la Décision, French Society for Operations Research and Decision Aiding] of the Association whose purpose is to bring together researchers and practitioners in the field.

The men and women in business or the media who use the term 'decision aiding' are not necessarily referring to a scientific activity. Conversely, when talking about decision aiding, OR-DA necessarily adopts a scientific approach to the issues involving decision-making. The following definition is commonly accepted and often quoted:

Decision Aiding is the activity of the man or women who, relying on clearly explicit, but not necessarily formalised models, helps a stakeholder to reach the elements of answers to the questions that he or she may ask during a decision making process. The elements should contribute to enlightening the decision, communicating about the decision, drafting recommendations, or promoting behaviour that would increase the coherence between the development of the decision making process on the one hand, and the goals and value systems that the stakeholder is serving, on the other (see Roy, 2005 and Roy 1999).

However, I cannot provide a definition for Operations Research because there is no widely accepted definition of the terms. The first definition was "Operations Research is what the Operations Research Company of New Jersey does." (For more details, readers may refer to Morse, Kimball, 1951, and Miller, Starr, 1969).

Several fields of applications of OR-DA, among others, can be found below:

- Assignment scheduling in a workshop or on a construction site

- Organising delivery or pick-up rounds

- Drafting service schedules or timetables in a transport company

- Dam water management 
- The assignment of agricultural plots of land to classes of risk

- The assignment of cancer cells to types of pathologies

- Routing communications within a telephone network

- Designing integrated circuits

- and more

Usefully, the reader's attention should be drawn to the following points. A person commonly called an analyst who practises decision aiding relies on working hypotheses and models to reach results based on scientific foundations. The results may have various outcomes: satisfactory or optimal solutions, compromise proposals, acknowledgment of incoherence or incompatibility, the statements of conclusions, or even recommendations. Importantly, one should always keep in mind that the purpose of DA is not to dictate the 'right decision' to the decision-maker. DA does not claim to uncover hidden truths. Decision aiding must be designed in such a way to mesh with the decision making process, thus enabling it to evolve in accordance with the goals of the different stakeholders and facilitate mutual communication.

\section{Two concrete examples}

a)Determination of the characteristics of a water supply network in an urban development zone

I had to deal with this problem at a time when robustness in OR-DA was not yet an issue. I was then the Scientific Director of SEMA (Metra International). René Loué, an engineer at the Road and Bridges Authority, asked me what I thought of how the Roads and Bridges engineers were using an operations research model to determine the structure and main characteristics of a water supply network for an urban development zone. The purpose of the very sophisticated model was to help define the optimal network that would meet a clearly defined demand for water, at the lowest cost, year after year over thirty years, in each neighbourhood of the zone. Based on the land use plan scheduled for each neighbourhood and the forecasts of household and business water consumption, the engineers had calculated the most probable volume of water that the network would have to supply, year after year, in each neighbourhood.

According to René Loué, the land use plans were likely change. How much the demand for water might change was also imperfectly known. Considering the imperfect knowledge and vague approximations in the modelling, René Loué felt that the optimum they had found was totally misleading. If, several years later, actual demand sharply exceeded what they had determined as the most probable demand (to which the network had been finely adjusted) in certain areas, the streets would have to be torn up to change water lines, install new lines and alter some other network features. On the other hand, if actual demand turned out to be much lower than the forecast probability, they would have made wasteful investments. Clearly the best way of formulating the problem was not to search for the characteristics of a network that, for a given demand (considered as known: year after year and neighbourhood per neighbourhood), would incur minimum investment and operational costs. The focus should rather be on gaining a grasp on what type of network would be the most likely to withstand a range of probable demands, taking account of the costs incurred by adjustments to actual demand. One, this meant that the approach could not be limited to a single scenario of the most probable demand, but had to introduce a range of plausible scenarios. Two, the model 
had to take account of what it would cost to adjust the initially designed network to the scenario that would actually happen.

b)Choosing the best bid after a call for tenders

I will deal with this type of problem by referring to a concrete case where I helped find a solution, some twenty years ago. At the time, the French Post had decided to fit its regional mail-sorting centres with package sorting machines, which were still far from perfect. So, the French Post issued an international call for tenders for the construction of a prototype. The call for tenders had very detailed specifications and limited the cost of the mass-produced machine to a "specified objective cost". The Post received fifteen bids.

Twelve criteria were used for the evaluation of each bid: prototype cost, stated deadline, the number of sorting directions, the number of sorted packages per hour, the rate of rejected or improperly sorted packages, sound levels, bag handling ease, occupied area, trust in the supplier to meet commitments, and three more technical criteria. Once each bid had been assessed by its 12 evaluation criteria, how should the best one be chosen? In this case, there was not one criterion to optimise. Considering the heterogeneity of the magnitudes serving for bid evaluation, designing a single criterion encompassing the 12 criteria was unrealistic. Regardless of the procedure selected to enlighten the choice, two aspects of the problem had to be taken into account:

i) Evaluating the bids on each criterion would inevitably comprise some uncertainty or arbitrariness

ii) The decision maker wanted some criteria to play a more important role than others

I will deal later with each of the two aspects that are not specific to this particular problem.

3. What is Robustness in OR-DA?

As can be guessed by the two above examples, vague approximations and areas of ignorance, which affect modelling as well as what is commonly called the data, limit the scientific approach in OR-DA. Several examples of vague approximations and areas of ignorance can be found below:

- Modelling involves simplifying reality: several different ways are possible and only one is selected.

- One particular piece of information whose value is debatable is taken into account as if it were certain

- A random variable is supposed Gaussian, mainly for the purpose of facilitating calculations

- Another source of vague approximations, which is often overlooked, involves the way of processing numbers as if they represented a quantity: in the calculation, a deadline that is twice as long will be counted as being twice as penalising, whereas it might actually be much more, or much less so.

- When modelling a system of (technical, economic, social) constraints, one often overlooks the uppermost limit that is allowable within the (pressure, budget, capacity) thresholds that mark (or will mark) the boundaries of the possible or the feasible in the future. 
- When there are multiple criteria, the metaphor of weight is generally used to differentiate the role that the different criteria must play. However, often the weights do not to refer to any objective reality, and the way they operate within the model is usually far from clear.

Importantly, the vague approximations and areas of ignorance (often referred to under the umbrella term 'uncertainties') have to be taken into account if useful answers are to be provided to the decision maker. Actually, not doing so may lead to misleading outcomes.

In OR-DA, robust is a qualifier that refers to an aptitude to withstand 'vague approximations' or 'areas of ignorance', to provide protection against deplorable impacts, such as results that are much worse that expected, or damage to properties to maintain.

The qualifier 'robust' applies to various aspects, i.e. solutions, conclusions, recommendations, methods. Robustness primarily involves a concern. The concern must be present throughout decision aiding, including at the time when the problem is formulated. This issue, which was raised at the end of section 2.a, is also highlighted in the following didactic example, which also underscores the share of subjectivity found in the concern for robustness.

Mrs. $D$ is wavering between three solutions for her trip from $A$ to $B$ :

- The subway: this solution involves a change on the line. However, as the trains run regularly, she can estimate travel time with a small margin of uncertainty.

- A bus and a train: the bus to the train station does not run very regularly. She also does not know the train timetable. However, the trains travel much faster than the subway does. Therefore, this solution is faster than the subway solution, but it might also take much longer if she has to wait for a bus and then for a train.

- A car: if there are no traffic jams, it is the fastest solution.

If the trip from $A$ to $B$ is a one-time event and if Mrs. $D$ absolutely does not want to be late, she will find that the subway solution, albeit longer, is the most robust. If, on the other hand, it is a regular trip and if Mrs. $D$ considers that slight delays (as long as they do not occur too often) are acceptable, she might feel that, on average, subway travel time will be much longer than the travel time on the two other means of transportation. So, the most robust solution has to be chosen from the two other solutions: even if traffic jams seldom occur, Mrs. $D$ may fear that they would have unacceptable outcomes, something that would be less likely with the bus-train solution. In this case, the latter would seem to her as the most robust.

In the paragraphs below, I will call the model on which decision aiding relies and the procedures for processing the model to reach results: formal representation (FR). I will call the context in which decisions will be made, executed and judged real life context (RLC).

The decisions for which decision aiding is performed will be implemented in a real life situation that, undeniably, will not strictly conform to the model on which the decision aiding relied. Furthermore, the decisions may be assessed in light of a value system, which may not necessarily be in perfect accordance with the system that was used to design and process the model. Therefore, seamless conformity between FR and RLC seems virtually impossible. This lack of conformity stems from extant vague approximations and areas of ignorance. The concern for robustness means that they have to be taken into account in the RF as best as possible; or the FR should consider at least those vague approximations or areas of ignorance that, if overlooked, would make decision aiding unable to protect the decision maker from any deplorable impacts. 
In a given decision making situation, if the analyst is to meet the concern for robustness properly, he or she should design the FR while taking account of two requirements that I will now explain.

4. Two requirements to meet the concern for robustness properly

First requirement: carefully inventory and properly take account of all frailty points in the FR.

By definition, a frailty point is a place in the model, or in a procedure processing the model where vague approximations or areas of ignorance can be found. Taking account of a frailty point means retaining either a single option or a series of possible options. An option means a particular, precise way of dealing with vague approximations or areas of ignorance. For instance, an option may be:

- A given value assigned to imperfectly known data, or to a poorly defined parameter

- A set of values setting the weight assigned to each of the relevant criteria

- A particular probability distribution to characterise a random variable

- A simplifying hypothesis making it possible to take account of a complex phenomenon

- A particular way of building a criterion to take account of a point of view

- A possible value assigned to a technical parameter involved in a processing procedure

- The choice of a solution among the solutions closest to an optimum solution

Given an identified frailty point, the analyst must ask him/herself whether - by retaining only one option for the frailty point - he or she is running the risk that outcomes of model processing might conceal impacts that the decision maker may deem undesirable. If this is true, the analyst should design a set of options likely to highlight the different eventualities. The set may be discrete (i.e. a few possible values, several sets of weights, two or three probability distributions, two or three plausible hypotheses) or continuous (i.e. intervals of possible values, families of probability distributions characterised by parameters with values within the given intervals).

Second requirement: design forms of answers likely to help the decision maker protect him/herself from undesirable impacts that may result from vague approximations or areas of ignorance in the FR.

The forms of answers have to take account of the decision maker's expectations of the said protection. Are there impact levels that he or she is ready to accept in certain circumstances? Are there other levels that he or she considers unacceptable; levels for which he or she wants protection, whatever the circumstances or cost? Depending on the real-life context, and because of the vague approximations and areas of ignorance in the FR, the decision maker's decision could, to some extent, reconcile his or her need for protection on the one hand, with his or her desire to optimise the performance criterion or criteria serving to evaluate the solution, on the other. The forms of the analyst's answers will only be truly useful for the decision maker if enough information is provided so that the latter can, in light of his or her own subjectivity, come to a decision given the two conflicting risks: 
- Finding him/herself poorly protected in the case of very poor performance revealing undesirable impacts

- Finding him/herself in a position whereby he or she forsakes any hope of good, or even very good performance

The way both requirements are taken into account in current journals prompts me to make two observations, which I will present briefly (for more details, see Roy, 2010a).

First observation: the frailty points that are usually taken into account solely relate to the 'data' whose purpose is to bring into play an aspect of RLC, which is called uncertain, into the FR.

This approach leads to designing a set of scenarios to describe all the realities that are likely to happen and that must be taken into account by the concern for robustness. A scenario is defined by a single option chosen with regard to each frailty point within a set of related options. The set of scenarios may be finite or infinite.

Therefore, a proper formulation of the problem present in section 2.a means involving other narratives, alongside the narrative of the most probable water volumes (neighbourhood per neighbourhood), with each plausible narrative making up a scenario. This example also shows that the relevant frailty points may also come from vague approximations and areas of ignorance, which are not necessarily related to the data on uncertain aspects of the RLC. Modelling water supply networks that will meet a given demand in the relevant zone involves various technical parameters. Several options for the value that will be assigned to each of the said parameters should be examined. The optimisation criterion making it possible to process the model can also be formulated in different ways. Consequently, using the term scenario to describe a choice of options relating to the said frailty points may be inappropriate when communicating with the decision maker, who may assign a different meaning to the term. To ensure that all the frailty points are considered without restriction, I find it preferable to replace the concept of scenario with the concept of version (of the formulation of the problem). A version is still defined as a combination of options taking account of all the relevant frailty points (for more details see Roy 2010a). However, I will be using the term 'scenario' in the rest of my paper. Importantly, the frailty points that may be found in the way the model is processed should not be overlooked. This may lead to taking account of all the procedures in set $P$ wherein each $p$ procedure is still defined by a precise option characterising the procedure for each of the frailty points contained therein, rather than merely considering a single processing procedure.

Second observation: most stakeholders are only interested in a single type of answer: find one (possibly several) solution(s) that can be qualified as 'robust'. To give the term meaning, they refer to a FR model where the performance of a solution is defined by a single criterion and they give preference to the performance of the solution in the worst-case scenario, to measure the robustness of the solution.

This type of answer relies on a fairly particular concept of a robust solution that fits within in a fairly restrictive schema that I will now address.

5. A framework schema to meet the concern for robustness

The schema may be defined by the three following characteristic traits.

Characteristic trait no. 1: in RF, the decisions, which have to be studied, are modelled as elements $a$ of a set $A$ of so-called feasible solutions. A single criterion $g$ associates a 
performance $g(a)$ with each element. This criterion clears the way for defining the optimal solution or solutions, regardless of any concern for robustness.

Set $A$ may be defined in comprehension by the set of solutions of a system of constraints, as can be seen in the example in 2.a. In this example, criterion $g$ is a cost criterion that has to be minimised. Set $A$ can also be defined in extension by a list, as is the case in the example in 2.b. However, in the latter example, it is not possible to characterise the performance of a bid by a single criterion to optimise.

Characteristic trait no. 2: a single measurement is defined to give meaning to the assertion "solution $x$ is as least as robust as solution $y$." This - and only this - measurement is involved when qualifying a solution as robust.

Three robustness measurements have been chiefly used since Kouvelis and Yu's famous work (1997). I present them below in the event criterion $g$ has to be minimised. On this subject, I will talk about cost (the cost being worth plus infinite for solution $x$, which is not feasible in scenario $s \in S$ ).

The first measurement relies on the notion of guaranteed cost. The guaranteed cost of solution $x$ is the highest cost for the solution in the model. Therefore, it is the highest value of $g(x)$ in every scenario. Taking this guaranteed cost as the definition of the robustness measurement is the same as admitting that solution $x$ is considered to be at least as robust as solution $y$, if, and only if, the guaranteed cost in $x$ is, at most, equal to the cost guaranteed in $y$.

The two other two robustness measurements rely on the concept of regret. One involves absolute regret and the other, relative regret. Solution $x$ having been selected, when the actual scenario is known, the decision maker may compare the $g(x)$ cost that he or she actually incurs in this scenario with the cost he or she would have incurred had the selected solution been the best in the scenario. Absolute regret is, by definition, the difference between the value of the $g(x)$ cost in the actual scenario and the cost incurred by the optimal solution in the scenario. Relative regret is defined by dividing absolute regret by the cost of solution $x$ in the relevant scenario. Admittedly, the decision maker does not know which scenario will happen, but the maximum value for (absolute or relative) regret within all the scenarios clears the way for associating what can be called guaranteed regret with each solution $x$. Taking guaranteed regret as the definition of the robustness measurement is the same as admitting that solution $x$ is considered at least as robust as solution $y$ if, and only if, the guaranteed regret in $x$ is, at most, equal to the guaranteed regret in $y$.

Characteristic trait no. 3: the single selected robustness measurement only involves the scenarios where it leads to the worst performance, or greatest regret, wherein what happens in the other scenarios does not play any role. Robust solutions are those that optimise the robustness measurement in every scenario.

Co-guaranteed robustness measurements, guaranteed (absolute or relative) regret, involve considering only what happens in the most unfavourable scenarios (in terms of performance or regret), regardless of what happens in the other scenarios, to determine whether solution $\mathrm{x}$ deserves to be qualified as robust. This is a very particular way of addressing the concern for robustness. It cannot correspond to the decision maker's picture of robustness. A solution thus qualified as robust may incur costs or cause regrets that are very mediocre in large number of scenarios. Basing the concern for robustness on the optimisation of a cost or a guaranteed regret means admitting that one should primarily protect oneself as best as possible against what might happen if the actual scenario is the worst (in terms of cost or regret) vis-à-vis the 
selected solution This way of addressing the concern for robustness is not appropriate for the decision maker unless he or she has a very strong aversion to risk.

Characteristic trait no.3 assigns a decisive role to the scenario (or scenarios) that determines the optimum of the robustness measurement in the definition of a robust solution (or solutions). The scenario (or scenarios) may be highly unlikely (for instance, corresponding to combinations of extreme options). Removing the scenario (or scenarios) from set $S$ may lead to defining very different solutions as robust. This dependence of the solution qualified as robust on the presence of this or that scenario in $S$ can be clearly seen in the case of the design of a water supply network (see 2.a), when all the scenarios were designed as was suggested in section 4 (see the end of the first observation).

6. Other ways of defining the concept of 'robust solutions'

The above considerations explain why several authors have suggested definitions of 'robust solutions' by avoiding characteristic trait no. 3 (see Aïssi and Roy, 2010, and Roy, 2010a). I have suggested three new robustness measurements ( $b w$-absolute robustness, $b w$-absolute deviation and $b w$-relative deviation). Their purpose is to allow the decision maker to improve the way he or she would like to reconcile the two conflicting risks (see the end of section 4). To do so, he or she must assign a value to two parameters, $b$ and $w ; b$ refers to a goal (in terms of cost or regret) that has to be reached or improved in the highest possible number of scenarios whereas $w$ sets the value of the worst cost or greatest regret that he or she would like to guarantee, regardless of which scenario actually happens $(w>b)$. The decision maker who agrees to assign a higher value to $w$ than to $w^{*}$, guaranteed by solution $x$ that optimises the measurement of the related cost or guaranteed regret, obviously runs the risk that solution $z$ (which is robust from the standpoint of the new selected measurement) may lead to a cost or regret higher than $w^{*}$, in a few scenarios. However, with solution $z$, the decision maker can trust that the costs or regrets will be better than what they would have been in solution $x$, in a very high number of scenarios (for more details, see Roy, 2010b).

Very few authors have attempted to define the robustness of a solution by involving several rather than a single robustness measurement (in other words, by avoiding characteristic trait no. 2). There are also very few authors that have attempted to address the concern for robustness, by avoiding characteristic trait no. 1. Readers may refer to the above-mentioned references for further details on both points.

Robustness can also be addressed by looking for types of answers that do not necessarily highlight solutions qualified as 'robust'. Providing the decision maker with so-called robust solutions is actually a very particular type of response, which does not always properly meet the second requirement (see section 4). I will now deal with a more general type of response.

\section{The concept of 'robust conclusions'}

I will begin by introducing several notations. Let us take scenario $s \in S$ that has a perfectly defined model $M(s)$ in FR, the said model translating the way that the decision aiding problem was formulated in the case where the real-life context corresponds to scenario $s$. In other words, $p \in P$ (see section 4 ) is a procedure considered for processing $M(s)$. The processing provides a set of outcomes $R(p, s)$ that may have various forms: optimal solution(s), uncontrolled solution(s), rejected solution(s), the establishment of an impossibility or inconsistency. The analyst relies on the said results to answer the decision maker's questions. For various reasons, the analyst may only focus on the outcomes involving pairs $(p, s)$ that belong to a given subset $Q$ of the Cartesian product $P \times S$. 
By definition, here the term conclusion means an assertion that takes account of a certain set of outcomes of the $R(p, s)$ type. This kind of assertion is called a robust conclusion when it includes the statement of the conditions governing the establishment of its validity. The conditions involve the relevant subset $Q$ of $P \times S \quad Q(P \times S)$ and the conditions and hypotheses that give meaning to, and warrant the assertion. Therefore, the robustness of a conclusion is contingent on the field of validity defined by the said conditions.

The most commonplace statement of robust conclusions is the following, "solution $x$ is robust, robust meaning that $x$ is a solution that optimises the measurement of robustness defined as follows (...) in all the outcomes $R(p, s)$ stemming from the relevant set $Q$." Here, the selected robustness measurement and the relevant set $Q$ define the field of validity of the robust conclusion.

There are less trivial forms of robust conclusions that do not refer to 'robust solution'. Several examples are given below.

"Except for the following procedures (...), all the $(p, s) \in Q$ pairs highlight the following solutions (...) as feasible with the following evaluations (...)."

$\forall(p, s) \in Q, x$ is a solution whose gap with the optimum solution never exceeds ... \%".

"There is no feasible solution that ensures that the following goals (...) (associated with different criteria) will be guaranteed, regardless of scenario $s \in S$."

"All the solutions $x_{1}, x_{2}, \ldots, x_{k}$ have the following properties (...), except perhaps in some scenarios that are deemed as highly unlikely."

I will briefly summarise the role that the concept of 'robust conclusions' has actually played in drafting recommendations in the example in 2.B, to highlight the practical contribution of 'robust conclusions'.

The set of feasible solutions was defined in extension by the 15 selected bids. Each bid was evaluated on 12 criteria. Six of the solutions could be initially eliminated. Therefore, the FR model relied on what is called the 'performance table', which provides the performance for each of the 9 solutions on each of the 12 criteria. To evaluate the performance on a concrete scale appropriate to each criterion, a group of experts of the French Post was set up. The frailty points related to the share of uncertainty or even arbitrariness, which might affect some of the evaluations, were inventoried. Basically, their impact could be taken into account by means of the concept of thresholds of indifference or preference, which do not have to be known to understand the rest of the example.

The selected processing procedure was ELECTRE IS. In the procedure, a set of weights characterising the relative importance that the decision maker wanted to assign to each criterion had to be defined. For some of the criteria, a veto threshold could be introduced. In this case, the decision maker was a committee comprising several Post Office Managers. The role that had to be assigned to each of the twelve criteria was not perceived in the same way by the Technical Manager, Personnel Manager, Sales \& Marketing Manager or Financial Manager. Therefore, weights and vetoes were the frailty points in the way the model was processed. 
To understand the meaning of the robust conclusions that were drawn, I do not have to go into the details of what set $Q$ of the relevant $(p, s)$ pairs covered. For each pair, the ELECTRE IS method highlighted a subset of solutions that seem the most satisfactory. The said subset had to be as a small as possible, with the following constraint: any solution that was not in the subset could be eliminated, as it was not as good as at least one of the solutions in the subset. The said subset (that here play the role of the $R(p, s)$ outcome) corresponded to what is called a kernel in graph theory. Consequently, the method associated a kernel with each pair $(p, s) \in Q$.

A summarised version of the robust conclusions that were drawn on these bases can be found below.

Conclusion no. 1 : bid $a_{1}$ was found in almost all the kernels (this assertion was completed by the list of $(p, s)$ pairs where $a_{1}$ was not in the matching kernel).

Conclusion no. 2: bids $a_{5}, a_{6}$ and $a_{8}$ could not be found in any kernel.

Conclusion no. 3: bid $a_{9}$ could be found in some kernels; when this was the case, lowering the veto power of criterion 12 was enough to remove $a_{9}$ from the kernel (resulting in $a_{1}$ becoming as good as $\left.a_{9}\right)$.

Conclusion no. 4: the three bids $a_{2}, a_{3}$ and $a_{4}$ formed a subset $C$ of actions, which were indifferent in each pairwise comparison, which could be found in a large number of kernels.

Saying that bids $a_{2}, a_{3}$ and $a_{4}$ were indifferent in each pairwise comparison meant that, with the available data at time, they could not be differentiated. Each bid of $C$ could be considered as at least as good as each of the two others, without being substantially better.

Conclusion no. 5: bid $a_{7}$ could be found in a large number of kernels; the actions of $C$ were usually incomparable with $a_{7}$.

Affirming this incomparability meant that, with the available data at the time, $a_{7}$ could not be considered as at least as good as any of the actions of $C$ and that, vice-versa, none of these actions could be considered as at least as good as $a_{7}$.

These robust conclusions (completed by an appropriate analysis) led to the following recommendations that were submitted to the Post General Directorate (for more details, readers may refer to Roy and Bouyssou, 1993, chapter 8).

a)In the case where no more than one prototype could be built, bid $a_{1}$ should be retained (see conclusion no. 1).

b)In the case where more than one prototype could be built, bids $a_{5}, a_{6}, a_{8}$ and $a_{9}$ should definitely be eliminated (see conclusions no. 2 and 3).

c)In the case where three prototypes could be built, bids $a_{1}$ and $a_{7}$ and one of the three bids of $C$ should be retained (see conclusions no. 4 and 5). The 12 relevant criteria did not clearly differentiate bids $a_{2}, a_{3}$ and $a_{4}$ (to do so, one could examine considerations that were not (or poorly) taken into account by this family of criteria). If $a_{1}$ was actually retained, selecting $a_{3}$ at the same time would be more justified that selecting $a_{2}$ or $a_{4}$ because unlike the two latter, $a_{3}$ was usually incomparable with $a_{1}$.

d)In the case where two prototypes could be built, it would appropriate, in addition to $a_{1}$, to chose either $a_{7}$, or one of the $C$ bids, as $a_{7}$ was hard to compare with $C$ bids, an option which basically brought the 'decision maker's' value system into play. 
8. Conclusion

To conclude, I would like to underscore two points.

$\left.1^{\circ}\right)$ In OR-DA, the notion of robustness has relatively tight connections with other notions such as flexibility, stability, reliability, adaptability, sensitivity, and sometimes even equity.

$2^{\circ}$ ) Meeting the concern for robustness supposes taking account of the eminently subjective way that the decision maker wants to protect him/herself from catastrophic performance without having to forsake the hope for good, or even very good performance. 


\section{References}

Aissi H., Roy B. (2010), Robustness in Multi-criteria Decision Aiding, in Trends in Multiple Criteria Decision Analysis, (Matthias Ehrgott, José Rui Figueira, Salvatore Greco), Springer, Science + Business Media, volume 142, pages 87-121

Billaut J.C., Moukrim A. and Sanlaville E., (2008), Flexibility and Robustness in Scheduling, Wiley-ISTE, New York

Kouvelis T., Yu G. (1997), Robust discrete optimization and its applications, Kluwer Academic Publishers, Boston

Miller D.W. and Starr M.K. (1969), Executive decisions and operations research, Englewood Cliffs, second edition, Prentice-Hall

Morse P.M. and Kimball G.E. (1951), Methods of operations research, Wiley, New York

Newsletter 2002-2008 of the European Working Group Multiple Criteria Decision Aiding No. 6-13. (see <http://www.inescc.pt/ ewgmcda/Articles.html>)

Rosenhead J., (2001), Robustness Analysis: Keeping your options open, in Rosenhead J., Mingers J., (Eds.), Rational Analysis for a Problematic World Revisited: Problem Structuring Methods for Complexity, Uncertainty and Conflicts, WileyChichester, pages 181-207

Roy B., (1999), Decision-aiding today: What should we expect?, in Tomas Gal, Theodor J. Stewart, Thomas Hanne (eds.), Multicriteria Decision Making - Advances in MCDM Models, Algorithms, Theory, and Applications, Kluwer Academic Publishers

Roy B., (2005), Paradigms and challenges, in Multiple Criteria Decision Analysis - State of the Art Surveys, (José Figueira, Salvatore Greco, Matthias Ehrgott), Springer, pages 3-24

Roy B., (2010a), To better respond to the robustness concern in decision aiding: Four proposals based on a twofold observation, in Handbook of Multicriteria Analysis, (Constantin Zopounidis, Panos M. Pardalos), Springer, pages 3-24

Roy B., (2010b), Robustness in operational research and decision aiding: A multi-faceted issue, European Journal of Operational Research 200, pages 629-638

Roy B., Bouyssou D., (1993), Aide multicritère à la décision : méthodes et cas, Economica, Paris. 